\title{
ASO Visual Abstract: Guide to Enhanced Recovery for Cancer Patients Undergoing Surgery-ERAS and Esophagectomy
}

\author{
Moorthy Krishna, MD, and Halliday Laura, MBChB, MSc \\ Imperial College, St. Mary's Hospital, London, UK
}

There is need for interventions to improve perioperative outcomes in patients with esophageal cancer. Enhanced Recovery After Surgery (ERAS) protocols are designed to accelerate recovery after surgery. Included in the summaries provided in our article (https://doi.org/10.1245/ s10434-021-10384-5). ERAS pathways are associated with a shorter length of stay and reduced incidence of respiratory complications following an esophagectomy.
Publisher's Note Springer Nature remains neutral with regard to jurisdictional claims in published maps and institutional affiliations.

\section{Summary of Items in Oesophagectomy ERAS ${ }^{\circledR}$ Pathway}

\begin{tabular}{|c|c|c|c|}
\hline Preadmission & Preoperative & Intraoperative & Postoperative \\
\hline $\begin{array}{l}\text { 1. Timing of surgery: } 3.6 \\
\text { weeks after neoadjuvant } \\
\text { chemotherapy or } 6-10 \\
\text { weeks after neoadjuvant } \\
\text { radiotherapy } \\
\text { 2. Nutritional assessment } \\
\text { and treatment based } \\
\text { on individual risk } \\
\text { 3. Smoking and } \\
\text { alcohol cessation } \\
\text { 4. Patient and family } \\
\text { counselling }\end{array}$ & $\begin{array}{l}\text { 1. Avoid routine use of } \\
\text { bowel preparation } \\
\text { 2. Avoid prolonged fast } \\
\text { (clear fluids allowed until } \\
2 \text { hours prior to surgery) } \\
\text { 3. Beta blockers for } \\
\text { patients with high } \\
\text { cardiac risk or those } \\
\text { on chronic beta blocker } \\
\text { treatment }\end{array}$ & $\begin{array}{l}\text { 1. Creation of gastric } \\
\text { conduit as first line option } \\
\text { 2. Two-field } \\
\text { lymphadenectomy for } \\
\text { T1b-T3/4 ACA in the } \\
\text { middle/lower third of } \\
\text { the oesophagus } \\
\text { 3. NG tube decompression } \\
\text { 4. Balanced crystalloid } \\
\text { fluid replacement } \\
\text { 5. Intermediate acting NMB } \\
\text { 6. Lung protective } \\
\text { ventilation strategies } \\
\text { 7. Avoid hypothermia }\end{array}$ & $\begin{array}{l}\text { 1. Consider thoracic } \\
\text { epidural as first line } \\
\text { analgesia } \\
\text { 2. Early enteral feeding } \\
\text { achieving full calorie } \\
\text { requirement by day } 3 \text { to } 6 \\
\text { 3. Early mobilization, with } \\
\text { defined daily targets } \\
\text { 4. Remove chest drains } \\
\text { when no evidence of air } \\
\text { or chyle leak } \\
\text { 5. Avoid positive fluid balance } \\
\text { 6. Target blood glucose } \\
\text { <10mmol// } \\
\text { 7. VTE prophylaxis for } \\
4 \text { weeks after surgery }\end{array}$ \\
\hline
\end{tabular}

(C) Society of Surgical Oncology 2022

M. Krishna, MD

e-mail: k.moorthy@imperial.ac.uk 